\title{
Viaje solo de ida. Gentrificación e intervención urbanística en Sevilla
}

Ibán Díaz-Parra. Universidad Nacional Autónoma de México, Ciudad de México, México.

RESUMEN | La gentrificación es un proceso de cierta complejidad, por sus múltiples facetas, lo que dificulta tanto las aproximaciones empíricas como su mensurabilidad. Este artículo propone una metodología de trabajo para la identificación de los sectores urbanos cuyo devenir se ajustaría a un proceso de gentrificación. La propuesta parte, por un lado, de la idoneidad de la utilización de la escala de ciudad para realizar una primera aproximación; y, por otro, de la necesidad de analizar la evolución histórica de la urbe para comprender este tipo de procesos. El trabajo se desarrolla en la ciudad de Sevilla en el marco del planeamiento general de 1987 y pretende reconocer la existencia de gentrificación o de indicios de la misma en sectores urbanos específicos, así como diferenciarla de dinámicas de distinta naturaleza.

PALABRAS CLAVE | gentrificación, segregación, planificación urbana, renovación urbana.

ABSTRACT | The many facets of gentrification make it a complex process, a condition that challenges both empirical approaches and the possibility of measuring it. This article pro $\neg$ poses a methodology for the identification of urban areas, the evolution of which respond to a gentrification process. On the one hand, this proposal is based on the use of the city scale as an initial approach. On the other hand, it is also based on the need to analyze the city's historical evolution in order to understand such processes. This methodology is applied to Seville in the context of the 1987 General Planning framework. It aims to recognize the existence of the process of gentrification in certain urban areas as well as to differentiate gentrification from processes of a different nature.

KEYWORDs | gentrification, segregation, urban planning, urban renewal.

Recibido el 16 de enero de 2013, aprobado el 20 de junio de 2013

E-mail: diaz@igg.unam.mx, rubeniban@yahoo.es

Correspondencia: Universidad Autónoma de México, Departamento de Geografía Social, Cubículo 4, Ciudad Universitaria, Coyoacán, 04510, México D. F. 


\section{Proposiciones sobre la transformación reciente de Sevilla}

La gentrificación se ha constituido en un tema de la investigación social en torno al cual se han desarrollado fuertes e interesantes debates desde la década de los setenta. En su mayor parte han tenido lugar en el ámbito anglosajón y solo de forma más reciente han empezado a cobrar peso en el mundo hispanohablante. En este contexto, el presente artículo pretende mostrar la adecuación del concepto a las transformaciones sociodemográficas recientes de los centros históricos de las ciudades españolas, frente a otras posibles interpretaciones de los indudables cambios que se han producido. Asimismo, pretende realizar una sencilla aproximación empírica al proceso. Para ello se desarrolla una metodología de análisis e indicadores específicos. Esta aproximación, no obstante, no obvia la necesidad de comprender la gentrificación como un proceso esencialmente histórico al mismo tiempo que espacial.

Utilizando el caso de la ciudad de Sevilla, se procederá a demostrar la adecuación del concepto a las últimas transformaciones de sus espacios centrales, un material que podrá ser comparado con otros casos en el futuro. Se ha tomado para ello el periodo comprendido entre los censos de 1981 y 2006, que cubre desde el primer Ayuntamiento democrático de la ciudad hasta el final de la última fase alcista del mercado inmobiliario en el Estado español, así como el ámbito de aplicación del planeamiento general urbanístico de 1987-2006.

La pregunta de partida del presente documento es: ¿se han producido procesos de gentrificación en Sevilla? Y para responderla debería atenderse al interrogante previo: ¿qué elementos concurren para hablar de un proceso de gentrificación?

Se parte de la evidencia de las fuertes transformaciones urbanísticas, sociales y demográficas acaecidas durante las últimas décadas en los sectores obreros de la ciudad histórica, comprendiendo por tal el recinto amurallado y sus arrabales. Así, tomando esto como punto de partida y descartando la existencia de gentrificación, podría argumentarse que la transformación fundamental es una repoblación de espacios que han sufrido un fuerte envejecimiento y abandono previos. Por lo tanto, la característica principal sería el incremento de la población de estos barrios, no el incremento del estatus. Por otro lado, la promoción social que hubiera podido producirse sería próxima a la media de la población sevillana durante el período de tiempo de estudio. Así, en el caso tratado, las transformaciones percibidas no consistirían tanto en procesos de gentrificación de sectores concretos, como en la promoción social generalizada de los habitantes, fruto de los cambios en su estructura sociolaboral.

Por el contrario, en este trabajo se afirma que los cambios de los antiguos sectores obreros del centro histórico coinciden con el modelo extraído del marco teórico - que se expondrá más adelante-, correspondiente a un proceso de gentrificación que se habría producido en el marco de las transformaciones urbanísticas propiciadas por el planeamiento general de 1987 para Sevilla y que se agotaría con el fin de la fase alcista del último ciclo económico en 2006.

Se concibe la ciudad como un espacio segregado, situación producida por el propio mercado de suelo y las diferencias sociolaborales. En este sentido, la característica fundamental del proceso de gentrificación es el cambio en la posición relativa de 
un sector en la estratificación social, que lo ha llevado desde una situación de escasa valorización, a situarse por encima de la media respecto de los indicadores de estatus. Esto es consecuencia de la sustitución de una población humilde por una de mayor estatus, un cambio social vinculado a la rehabilitación del espacio público y privado.

De acuerdo con la definición implícita en el marco teórico, la transformación social se produce de forma simultánea a la rehabilitación del parque de viviendas y la mejora de las condiciones de habitabilidad general. Asimismo, se excluyen de la definición adoptada los procesos de promoción basados en nuevos desarrollos residenciales sobre suelos que con anterioridad tuviesen otros usos, lo que podría denominarse "residencialización".

La proposición aquí realizada se aceptará mediante la identificación de una serie de sectores urbanos que coinciden con las características de un proceso de gentrificación, habiéndose descartado la repoblación de los mismos. Los barrios gentrificados tendrían una promoción social relativa, lo que descartaría la transformación como mero reflejo de los cambios en la estructura sociolaboral, al mismo tiempo que concurrirían otras características fundamentales del proceso. Por otro lado, la hipótesis de la repoblación se descartaría mediante la comprobación de las variaciones de población en el período en el que se produce la promoción social, así como por la evidencia de entradas y salidas de población atípicamente voluminosas.

Las fuentes estadísticas utilizadas para el establecimiento empírico de los hechos son el Censo de Población y Vivienda y el Padrón Municipal. Los datos se tomaron del Instituto Nacional de Estadística para los censos de 1981, 1991 y 2001 a escala de sección censal, agrupadas estas en unidades espaciales denominadas barrios. Los datos solicitados cubren aspectos sociolaborales y demográficos referidos a la población, y otros sobre el estado y características del parque de viviendas. Esta fuente, consultada hasta 2001 a causa de la periodicidad decenal de los censos, se ha complementado con el acceso al Padrón Municipal de Sevilla para el año 2006.

Para obtener la distribución de los casos de acoso a inquilinos, se ha recurrido a los archivos de la Oficina Técnica de Atención al Inquilino en Situación de Abuso (Otainsa), perteneciente a la Gerencia de Urbanismo del Ayuntamiento de Sevilla. Los datos sobre casos de acoso proporcionan información sobre los contenidos de los mencionados archivos, pero en ningún caso sobre el total de los casos de acoso o los desalojos acaecidos en la ciudad.

\section{Debate teórico sobre la gentrificación}

Smith y Williams iniciaban el volumen colectivo que coordinaron en 1986 sobre gentrificación, manejando varias definiciones para el término. La definición del Oxford American Dictionary resultaba en principio bastante esclarecedora: un "movimiento de familias de clase media en áreas urbanas que producen un incremento de los valores de la propiedad y que tiene como efecto secundario la expulsión de las familias más pobres" (Smith \& Williams, 1986, p. 1). Esta es una definición que coincide en gran medida con el sentido que le dio Glass al término en 1964 -autora a la que se le atribuye la primera referencia al vocablo-, como proceso vinculado a espacios urbanos históricamente obreros que implica la renovación y mejora del 
estatus de las viviendas, el desplazamiento de sus ocupantes originales y su sustitución por efectivos de clase media.

Siendo un concepto polémico, la gentrificación cuenta con firmes defensores y acérrimos enemigos. Este debate podría interpretarse como una traducción a la comunidad científica de los intereses enfrentados en torno al proceso. Por un lado, una administración pública y un capital inmobiliario claramente interesados en el desarrollo de estos procesos en sus centros urbanos, así como una clase media gentrificadora que prefiere verse a sí misma en el rol de "repobladora" o "pionera" antes que "colonizadora", en el sentido más negativo de la palabra; por otro lado, grupos que se ven desplazados fuera de estas zonas (Smith, 1996), así como voces críticas en las ciencias sociales, el periodismo o la política. De esta forma, las transformaciones sociales y urbanísticas que algunos autores interpretan como gentrificación, son identificadas, en aproximaciones con un carácter menos crítico, como "residencialización". Con este término se alude a una repoblación de un vacío demográfico previo o una transformación basada en el incremento de la carga residencial (Bromley, Tallon \& Thomas, 2005; o discusión en Lees, Slater \& Wyly, 2008).

La creación de nuevos barrios puede confundirse con la rehabilitación y la promoción social propias de la gentrificación. Para evitarlo, Neil Smith (1982) realizaba una distinción teórica entre este concepto y el de redesarrollo ${ }^{1}$. En un principio, Smith entiende como una parte fundamental de la gentrificación la rehabilitación de un determinado hábitat urbano, y no su demolición y la construcción de un nuevo hábitat ex novo. No obstante, en los artículos publicados en la última década, Smith cambia su opinión y flexibiliza la definición, incluyendo algunos de estos procesos de redesarrollo. Redesarrollo y gentrificación son conceptos diferentes pero no excluyentes; no obstante, existen autores que hablan de una new build gentrification a propósito de procesos de redesarrollo de espacios que anteriormente no habían estado habitados, por ejemplo enclaves industriales o portuarios (Slater, 2009, p. 294). En estos casos no existiría una sustitución de población, debido a que no habrían sido previamente habitados, y sería incorrecto hablar de gentrificación tal y como aquí se define el concepto. Otra cosa es que, como argumentan Davison y Lees (2010), las operaciones de redesarrollo de grandes sectores no residenciales generen gentrificación en los barrios colindantes.

La cuestión fundamental es que, para zonas residenciales, al utilizar los términos 'repoblación' o 'residencialización' se niega la existencia de desplazamiento, siendo esta una de las principales características del proceso (Smith, 1996). No obstante, muchos autores resaltan la escasa demostración empírica del desplazamiento forzoso de la población, a pesar de las tres décadas de estudios sobre este tipo de procesos (por ejemplo, Freeman, 2008, respondiendo a Slater, 2006). En este sentido, Hamnett (2003) argumenta que gran parte de la promoción social

1 "Por gentrificación entiendo el proceso por el cual vecindarios de clase obrera son rehabilitados por clases medias, propietarios y profesionales de la construcción. Hago una distinción teórica entre gentrificación y redesarrollo. Redesarrollo implica no la rehabilitación de estructuras viejas, sino la construcción de nueva edificación sobre suelos que habían sido desarrollados previamente" (Smith, 1982, p. 139). 
asociada a la gentrificación (entendida como profesionalización de los perfiles sociolaborales) es un proceso estructural en el que los obreros, por jubilación, muerte, etcétera, van siendo sustituidos por profesionales y técnicos, subiendo la proporción de estos incluso en los barrios más pobres. Así, el reemplazo sería resultado de los cambios a largo plazo en la estructura ocupacional. Tal posición puede identificarse con los trabajos más recientes de Hamnett (2003 y 2009) y Butler (2007), y encuentra eco en el ámbito latinoamericano, por ejemplo en Sabatina, Sarella y Vásquez (2012).

Para reconocer o descartar la existencia de gentrificación, deberían identificarse las características del gentrificador, del colectivo potencialmente desplazado y del espacio gentrificable. Para empezar, el sujeto gentrificador típico es definido por David Ley (1996) como un hogar de clase media, habitualmente sin hijos, a menudo de una persona soltera, principalmente por debajo de los 35 ańos de edad, empleada en el sector de servicios avanzados, con elevado nivel de formación, que recibe salarios moderados o elevados a pesar de su edad, y con pequeńas proporciones de minorías raciales o nacionales (p. 35). La emergencia de este grupo con elevados salarios se asocia a los cambios en la estructura económica, a la deslocalización industrial y a la emergencia de las ciudades como centros de negocios. La ubicación central del sector financiero, de los servicios avanzados y las oficinas en general, ofrece una explicación al desplazamiento de las nuevas clases medias -por la proximidad a su centro de trabajo-, algo repetido hasta la saciedad por Ley (1983), Beauregard (1986) o Smith (1996).

Por otro lado, la mayor parte de los estudios se refieren a la población desplazada como clase obrera, sin llegar a profundizar excesivamente en las características de este grupo en los distintos casos estudiados, algo sobre lo que se lamentan Slater (2006) o Wacquant (2008). Siguiendo a Beauregard (1986, p. 49), la población más proclive a ser desplazada es aquella que vive en edificios baratos pero deseables arquitectónicamente y cercanos al distrito de negocios. Muchos son marginales en el mercado de trabajo o están fuera de él: desempleados, jóvenes trabajadores, ancianos, personas dependientes de subsidios e individuos cercanos a la línea de la pobreza, descripción que coincide con las variables utilizadas por Atkinson (2000) en su estudio sobre el desplazamiento en Londres.

Una característica especialmente relevante que suele encontrarse en la población vulnerable de este tipo de zonas es el envejecimiento (Fernández Salinas, 2003). Se trata de barrios antiguos colonizados mucho tiempo atrás y en los que la falta de inversión no ha atraído masivamente a nuevas oleadas de inmigrantes. Puede haberse estado introduciendo población de forma constante pero no masiva, de tal manera que la tasa de envejecimiento del barrio gentrificable tiende a ser muy elevada. Finalmente, la consecuencia lógica de un vecindario envejecido y empobrecido es el submantenimiento del mismo por parte de los propietarios, vinculándose el cambio social al cambio del medio físico (Smith, 1996).

En resumen, las transformaciones que podrían esperarse en un sector urbano que se gentrifica serían las expuestas en el Cuadro 1. 
CUADRo 1 | Modelo de gentrificación extraído del marco teórico

\begin{tabular}{|c|c|c|c|}
\hline & $\begin{array}{c}\text { ESPACIO } \\
\text { GENTRIFICADO }\end{array}$ & $\begin{array}{c}\text { ESPACIO } \\
\text { GENTRIFICABLE }\end{array}$ & EFECTO ESPERADO \\
\hline \multirow{2}{*}{$\begin{array}{l}\text { Condición } \\
\text { sociolaboral }\end{array}$} & $\begin{array}{l}\text { Asalariados en trabajos } \\
\text { de elevada cualificación }\end{array}$ & $\begin{array}{l}\text { Asalariados en trabajos } \\
\text { de baja cualificación }\end{array}$ & $\begin{array}{l}\text { Incremento de la ratio sociolaboral } \\
\text { entre profesionales y técnicos y } \\
\text { trabajadores manuales }\end{array}$ \\
\hline & Población joven & Población envejecida & Rejuvenecimiento \\
\hline $\begin{array}{l}\text { Espacio } \\
\text { privado }\end{array}$ & $\begin{array}{l}\text { Viviendas en buen estado } \\
\text { y con precios elevados }\end{array}$ & $\begin{array}{l}\text { Viviendas en mal estado } \\
\text { y devaluadas }\end{array}$ & $\begin{array}{l}\text { Reducción del número de vivien- } \\
\text { das en mal estado }\end{array}$ \\
\hline \multirow{2}{*}{ Sucesión } & \multirow{2}{*}{$\begin{array}{l}\text { Hogares de estableci- } \\
\text { miento reciente }\end{array}$} & \multirow{2}{*}{$\begin{array}{l}\text { Indicios de desplaza- } \\
\text { miento de población } \\
\text { con escasos recursos }\end{array}$} & $\begin{array}{l}\text { Elevada proporción de hogares } \\
\text { establecidos en las últimas décadas. }\end{array}$ \\
\hline & & & $\begin{array}{l}\text { Elevada proporción de acoso/des- } \\
\text { plazamiento. }\end{array}$ \\
\hline
\end{tabular}

FUENTE ELABORACIÓN PROPIA

\section{La transformación de Sevilla en el marco del planeamiento de 1987}

La gentrificación opera sobre una estructura socioespacial previa. En este artículo se propone que los procesos de gentrificación han transformado esta estructura, para el caso sevillano, en el ámbito temporal de aplicación del planeamiento general de 1987-2006.

Con objeto de hacer más accesible el contenido de este artículo, en la Figura 1 se han representado las unidades espaciales utilizadas, indicando el nombre de los sectores urbanos mencionados.

\section{La estructura socioespacial previa de Sevilla}

De forma muy sintética, la diferenciación social histórica de la ciudad adoptaba la geografía que a continuación se detalla, a su entrada en la década de los ochenta.

En primer lugar, tenía una componente norte-sur con su origen en las características propias del emplazamiento de la urbe sevillana, así como en su desarrollo urbano primitivo. Esta polarización comenzó en el propio centro histórico, que en su sector sur ha acogido tradicionalmente las funciones rectoras, el primer centro comercial y financiero de la ciudad y la vivienda burguesa, mientras que el norte se consolidó a finales del xix como espacio industrial y obrero. Este carácter proletario era similar al de los principales arrabales históricos de la ciudad, especialmente San Bernardo y Triana. Durante las décadas del desarrollismo, estos barrios populares pasaron por un fuerte proceso de decadencia urbanística y social, con operaciones de renovación urbana muy puntuales en el extremo sur del arrabal de Triana y en San Julián.

Junto a la anterior había una componente este-oeste, fundamentada en la existencia de dos barreras urbanas, como serían la línea del ferrocarril Sevilla-Cádiz y la Ronda del Tamarguillo, que separaban operaciones de ensanche burgués de la primera mitad del siglo xx, de sectores de autoconstrucción y de bloques de pisos en manzana de carácter obrero. 
FIGURA 1 | Barrios de Sevilla

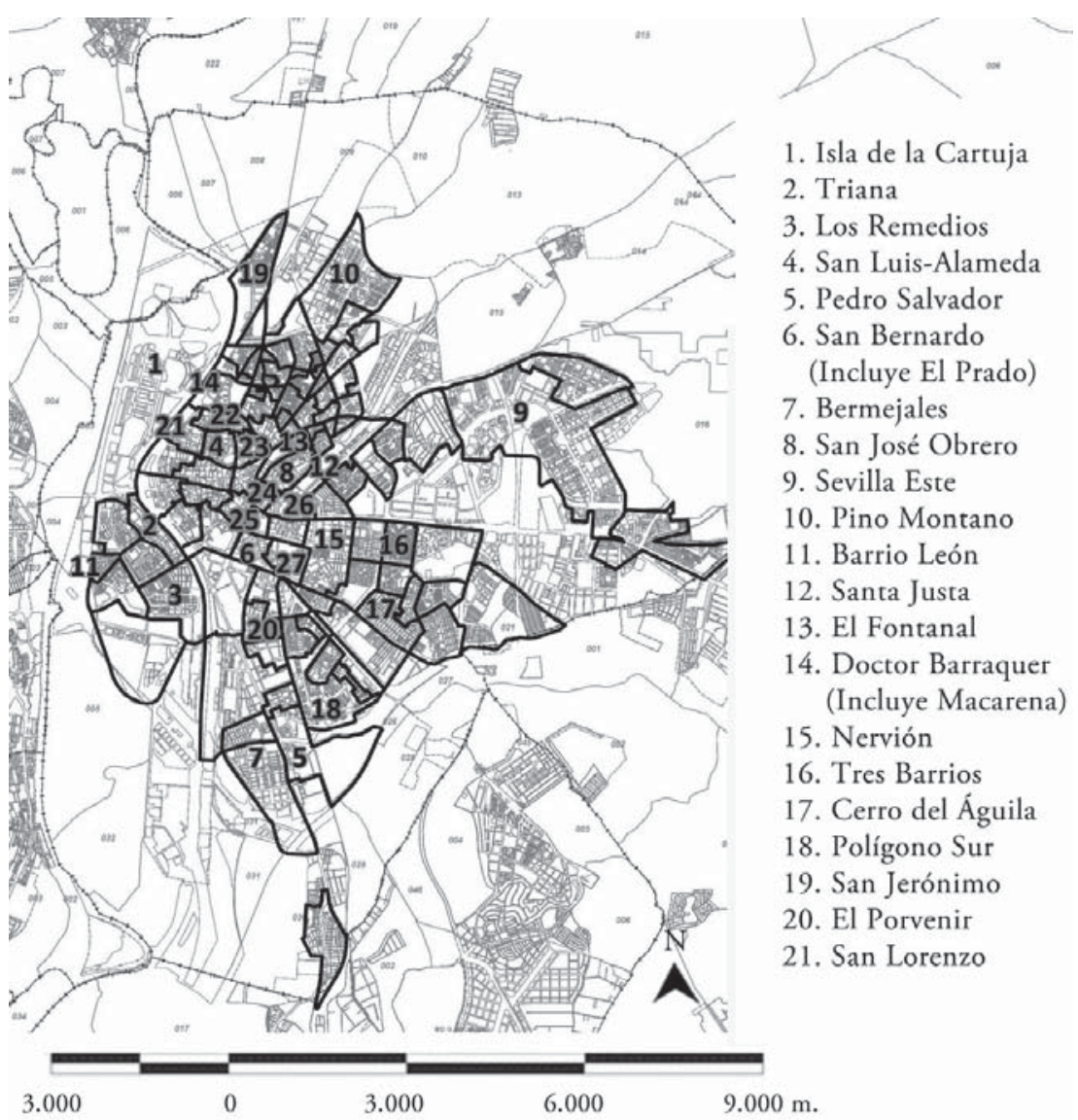

FUENTE ELABORACIÓN PROPIA

Este esquema básico se habría reproducido en función de las elecciones de los agentes económicos y la racionalización por parte del Estado de las dinámicas del mercado a la hora de situar las principales promociones de iniciativa pública, a partir de la década de los cincuenta.

Existe una amplia bibliografía que hace referencia a estas cuestiones, entre las que destacan las obras de González Dorado (1975), Marín de Terán (1980), Cruz Villalón (1986), Martín García (1996) y García Bernal (2005).

Sobre este escenario intervino el documento de ordenación urbana de 1987. Dicho plan se enfrentaba a un trazado ferroviario problemático, un sistema de carreteras insuficiente para las dimensiones que había adquirido la ciudad, una escasa integración con el río y un centro histórico en decadencia avanzada. A esto se le sumaban un notable déficit de equipamientos y elevadas densidades de población que afectaban tanto a las barriadas obreras como a gran parte de las destinadas a clases medias y acomodadas (Fernández, 1993, p. 388).

Las transformaciones relevantes para este trabajo que el planeamiento operó sobre la ciudad son las que se exponen a continuación. 


\section{Sistemas viario y ferroviario}

Una de las determinaciones más importantes fue la reestructuración del tejido ferroviario. Para recomponer la ciudad a partir de la transformación del trazado de su ferrocarril, se desarrollaron tres operaciones fundamentales:

- La recuperación de la perdida relación ciudad-río, con la supresión del ramal entre San Jerónimo y Plaza de Armas y la remodelación de Torneo.

- El redesarrollo del entorno de San Bernardo sobre la base del soterramiento de la línea del ferrocarril.

- La localización de la nueva estación de Santa Justa, con la consiguiente modificación de su entorno urbano.

El soterramiento desde Santa Justa a la calle Bogotá permitió la completa regeneración del denominado "cuadrilátero de San Bernardo". El levantamiento de las estructuras ferroviarias sobre Torneo, por su parte, dio lugar a una nueva avenida y a la reestructuración del frente del río (Gerencia de Urbanismo, 1987).

Estas operaciones, junto con importantes intervenciones sobre la estructura viaria radioconcéntrica de la ciudad, propiciaron la transformación de varios sectores históricos. El soterramiento del ferrocarril permitió la remodelación del corredor Santa Justa-San Bernardo, con la recalificación de suelos industriales o dedicados a infraestructuras ferroviarias, así como la remodelación de los degradados arrabales de San Bernardo y La Calzada. La supresión del ramal que conectaba la estación de Córdoba con San Jerónimo, el establecimiento de nuevos vados y la recuperación de un trazado del río próximo al original, junto con la urbanización de la Isla de la Cartuja (recinto de la Exposición Universal de 1992 y actualmente parque tecnológico y temático), afectaron radicalmente tanto la centralidad del norte del recinto intramuros como a los arrabales de Macarena y Triana.

\section{Intervención sobre el centro histórico y sus arrabales}

Resulta de interés resaltar el impacto de la reestructuración urbana sobre los arrabales de La Calzada y, sobre todo, San Bernardo.

Un elemento importante del Plan General de Ordenación Urbana de Sevilla (PGOU) (Gerencia de Urbanismo, 1987) era aceptar y gestionar el hecho de que las funciones centrales fueran a dejar de ser patrimonio exclusivo de la mitad sur del centro histórico. Así, la centralidad se difundiría en parte alrededor del casco, principalmente sobre San Bernardo y el sector de La Buhaira. El pujante espacio central de Nervión, con base en la avenida San Francisco Javier, se concebía como una difusión del centro comercial y financiero, dificultada por la presencia del arrabal y la barrera ferroviaria. Y continúa el Plan: "no casualmente, esta localización, devenida plenamente central en términos relativos con el crecimiento de la ciudad, constituye un área irresuelta, cuya remodelación adquiere un papel trascendental para el conjunto de aquella" (Ibídem, p. 35). El Plan veía en esta pieza un gran potencial articulador entre el centro y la periferia este. En conclusión, con la intervención prevista, "se inicia quizás, con mayor rotundidad que en ningún otro lugar de la ciudad, el proceso de transformación interna, sobre sí misma, que Sevilla requiere como alternativa coherente y actual a una innecesaria extensión” (Id.). 
Se preveía que una nueva vía norte-sur tuviera también un impacto positivo en el barrio de La Calzada. En consonancia, se propuso la recuperación de parte de la trama primitiva y la sustitución y transformación, allí donde se consideraba impensable la restitución. En teoría, la ordenación propuesta devolvería la imagen tradicional al barrio. La realidad posterior fue radicalmente distinta. El arrabal sería erradicado mediante una operación de renovación urbana. La mayor parte de este barrio se dedicó a un nuevo desarrollo en grandes manzanas de bloques exentos - edificaciones separadas de todos los lindes del terreno - sin ninguna relación con su morfología anterior. Para San Bernardo se propuso el mantenimiento de su arquitectura protegiendo el patrimonio edificado y algunas modificaciones en su estructura, como el retoque de sus bordes para mejorar la accesibilidad de la pieza, el desarrollo de un nuevo espacio verde y nuevas edificaciones sobre las infraestructuras obsoletas asociadas al ferrocarril. Esto por medio de varios planes de reforma interior, parte de los cuales están aún en proceso de desarrollo.

A pesar de la pérdida relativa de peso que el planeamiento general admitía y se proponía gestionar, el centro histórico debía seguir teniendo una descomunal importancia para el conjunto de la ciudad. Los objetivos para este gran espacio urbano pasaban por su "revitalización residencial", para lo que eran necesarias su regeneración urbanística y la conservación de su patrimonio físico. Al mismo tiempo, el Plan mostraba su voluntad de romper con el inmovilismo que imponía el planeamiento previo, excesivamente proteccionista para con el espacio histórico (Ibídem, p. 42).

Gran parte de las determinaciones y del planeamiento de desarrollo para el centro histórico quedarían supeditados a la aprobación del Plan Especial de Protección del Conjunto Histórico de Sevilla (véase Gerencia de Urbanismo, 1994). De hecho, los planes de reforma interior para San Luis (centro histórico norte) y San Bernardo serían tramitados de urgencia, integrados dentro de planes especiales de protección a los que se añadía un catálogo de la edificación, ejecutándose en su mayor parte en la segunda mitad de la década de los noventa. En el caso de San Luis, las operaciones de reforma de su trazado se desarrollarían de forma simultánea a la implementación de la iniciativa europea Urban (García Jaén, 1998).

\section{Los nuevos desarrollos y redesarrollos}

El planeamiento general urbanístico de 1987 estaba volcado en la ciudad consolidada y no preveía una expansión considerable para Sevilla. Dentro de esta realidad, fueron dos los únicos nuevos sectores urbanos con fuerte carga residencial que se desarrollaron: Los Bermejales y Sevilla Este (Figura 2). Además de estos dos grandes sectores residenciales, se estableció la ocupación residencial de terrenos intersticiales, con cuyo desarrollo se pretendía alcanzar una mayor continuidad y estructuración de la periferia obrera de la ciudad, entre los barrios de autoconstrucción al este del Cerro del Águila y entre Pino Montano y San Jerónimo. Un tercer grupo lo formarían los nuevos desarrollos fruto de la recalificación de terrenos no residenciales con una ubicación progresivamente céntrica, principalmente en torno al centro histórico, dentro de los cuales destacan la liberación de terrenos por el cambio del trazado del ferrocarril, pero también la recalificación del suelo anteriormente ocupado por viejas fábricas obsoletas o relocalizadas. Entre las nuevas 
oportunidades contempladas por el Plan que dieron lugar a zonas muy dinámicas respecto de la construcción de viviendas en los ańos noventa, la más destacable fue la zona al este del centro histórico, que incluía la rehabilitación de San Bernardo y el nuevo desarrollo de La Buhaira. También fueron destacables las nuevas construcciones en el entorno de la estación de ferrocarril de Santa Justa y en el espacio entre el arrabal de la Macarena y el río.

\section{Figura 2 La transformación de Sevilla en el marco del Plan General de Ordenación Urbana de Sevilla 1987}

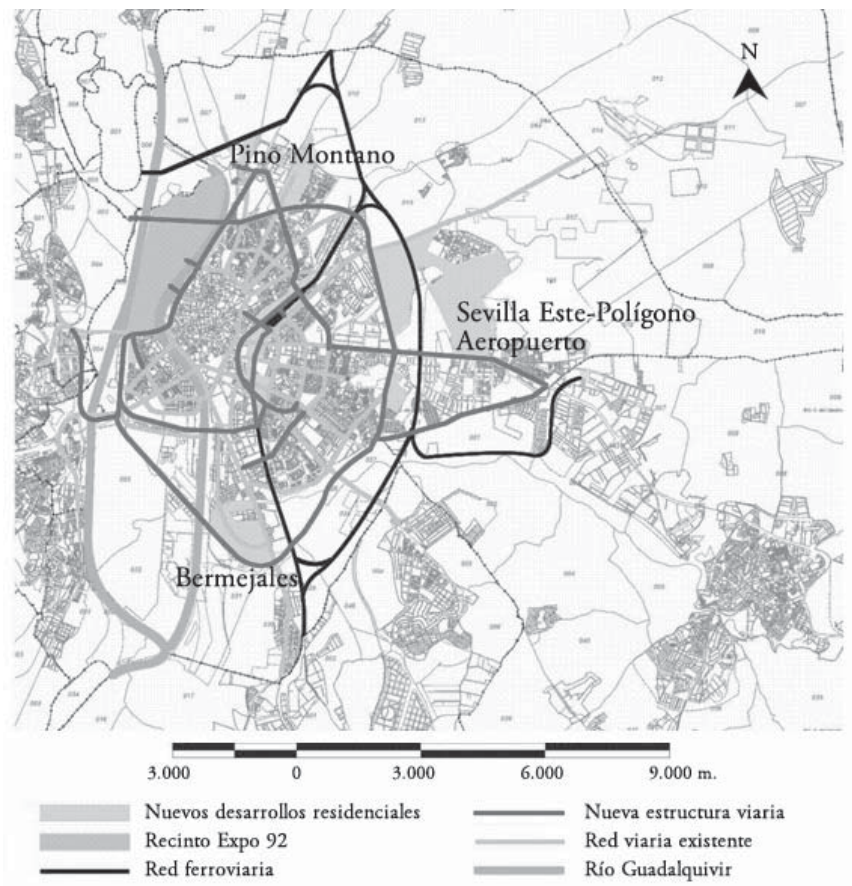

FUENTE ELABORACIÓN PROPIA

\section{Análisis sociodemográfico}

\section{Cambio sociolaboral}

La ratio sociolaboral global ${ }^{2}$ para Sevilla se habría incrementado en 105 puntos en el período comprendido entre 1981 y 2001. Se comprueba cómo los trabajadores de cuello azul reducen su peso en la población total, un descenso que tiene su reverso en el incremento de profesionales, técnicos y asimilados. No obstante, esta transformación del perfil sociolaboral no ha sido uniforme en el conjunto de la ciudad. Para mostrar este comportamiento disímil, se ha representado la ratio sociolaboral en tramos de la desviación típica en torno la media de la serie, para los ańos 1981 y 2001 (Figura 3).

A primera vista, se aprecia que para ambos años de referencia, la mayor parte de los barrios se encuentra entre la media y una desviación típica por debajo de la media. En segundo lugar, se hace evidente el incremento del número de barrios situados por encima de la media. 
FIGURA 3 | Ratio social. Desviación respecto de la media

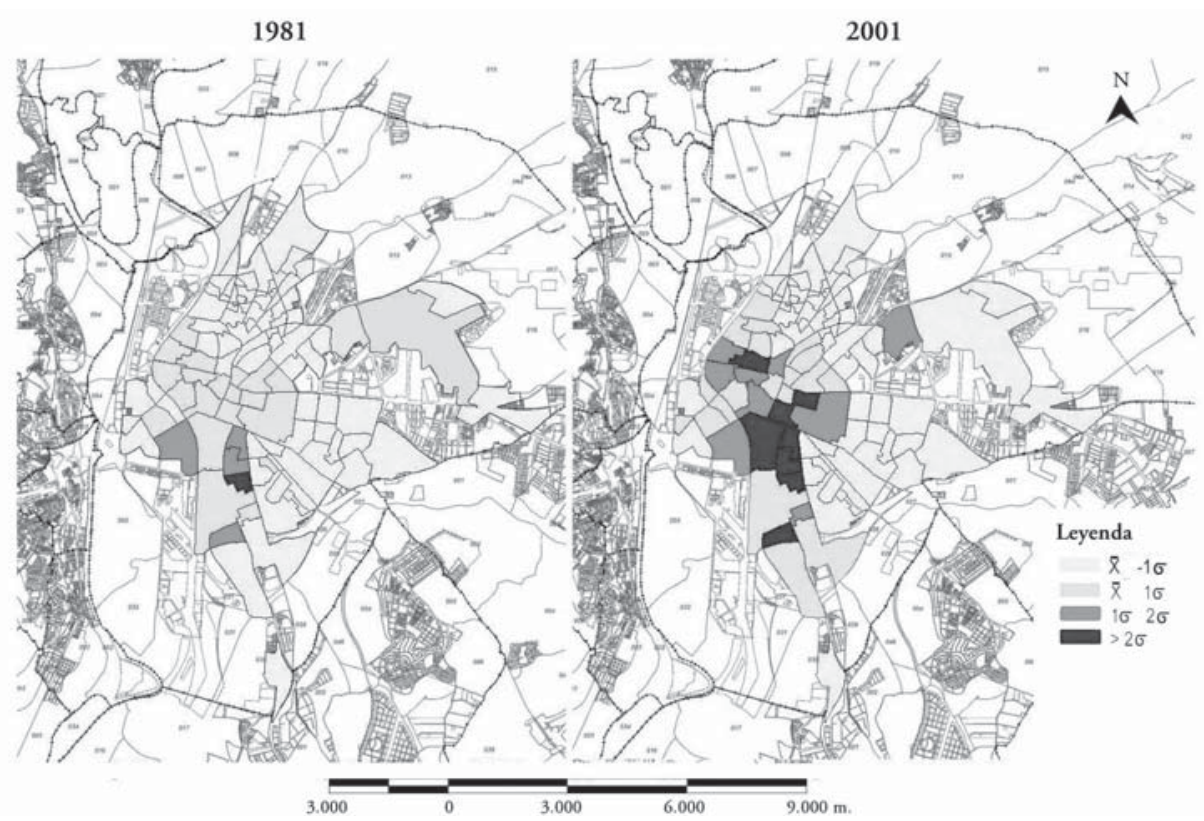

FUENTE ELABORACIÓN PROPIA A PARTIR DE LOS CENSOS DE POBLACIÓN Y VIVIENDA DE I 98 I Y 200 I

Entre 1981 y 2001 se produjo una notable promoción social de la ciudad. Fueron, sin embargo, los sectores más privilegiados los promocionados en mayor medida: es el caso de los ensanches este y sur, Nervión y Porvenir, y de la mitad sur del casco. Por otro lado, las zonas por debajo de la media, la periferia obrera estable de la ciudad, fueron las que menos se promocionaron, considerando este indicador. Sin embargo, el mayor interés en este caso está en aquellos ámbitos que partían de un valor bajo y se han visto promocionados por encima de la media de la ciudad.

Las unidades con una promoción social relativa son numerosas y mantienen la continuidad espacial con las ya privilegiadas en 1981, reproduciendo así el patrón previo. Los sectores que se han visto promocionados de esta manera son los siguientes:

- El entorno de Santa Justa; en concreto, las unidades espaciales de San José Obrero y El Fontanal. El resto del corredor Santa Justa-San Bernardo, donde se localizan los arrabales de La Calzada, San Roque y San Bernardo, se encontraba ya en 1981 ligeramente por encima de la media, aunque también se ven notablemente promocionados, en especial San Bernardo.

- El frente de expansión al sur de la ciudad, donde se produce en este periodo el desarrollo de Los Bermejales.

- Doctor Barraquer, que incluye el arrabal de la Macarena, así como un buen número de promociones residenciales nuevas propiciadas por el desmantelamiento del ferrocarril.

- El cuadrante noreste del centro histórico (excepción hecha de San Julián).

- Las dos unidades espaciales que comprenden el arrabal de Triana y el Barrio León. 


\section{Cambio demográfico}

La comparación de los efectivos de población de Sevilla entre 1981 y 2006 arroja un saldo positivo de 60.472 habitantes. En los períodos 1981-1986, 1991-1996 y 1996-2001, la población experimenta ligerísimos incrementos, siendo el período de mayor crecimiento el situado entre 1986 y 1991, fecha a partir de la cual la población sevillana sufre una notable desaceleración. Esto es así de tal forma que en el período 2001-2006 el saldo pasa a ser negativo.

Íntimamente vinculado al estancamiento de la población se encuentra el incremento del índice de vejez. El envejecimiento y la consiguiente reducción del crecimiento vegetativo, sin llegar a ser negativo, son una de las razones de este estancamiento, siendo su causa fundamental el saldo migratorio negativo. De esta forma, el índice de vejez habría pasado de un prudente $8,66 \%$ a superar el $14 \%$ en la presente década.

\section{FIGURA 4 | Crecimiento real de la población por barrios 1981-2006}

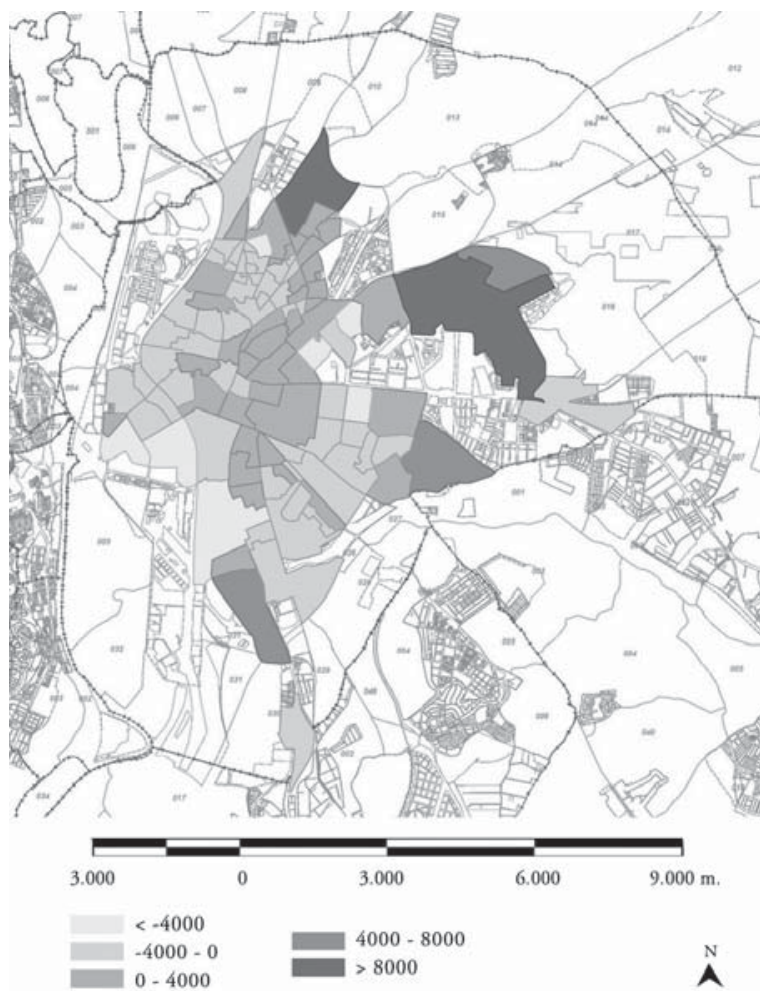

FUENTE ELABORACIÓN PROPIA A PARTIR DE LOS CENSOS DE POBLACIÓN Y VIVIENDA DE I98I, I99I Y 2OOI, Y PADRÓN MUNICIPAL DE HABITANTES DE SEVILLA

Respecto del crecimiento real de la población, los barrios ofrecen variaciones moderadas entre 1981 y 2006 ; la gran mayoría varía entre 0 y -4.000 o entre 0 y 4.000 individuos (Figura 4). En la primera periferia obrera de la ciudad, parece predominar el primer caso, mientras que los sectores obreros más periféricos, y no pocos enclaves históricos, se encuentran en el segundo caso. 
Los dos barrios con mayor crecimiento real serían, por este orden, Sevilla Este y Pino Montano, que han visto incrementos por encima de los 8.000 individuos. A gran distancia se encontrarían los otros dos frentes de expansión activos de la ciudad, Los Bermejales y Palmete, con incrementos en torno a los 6.000 individuos.

En las zonas en que se ha registrado un profundo cambio social, este ha venido unido a un intenso incremento de la población solo en una parte de ellas. El caso más evidente es el de Bermejales. Los sectores atravesados por el ramal del ferrocarril soterrado entre San Bernardo y Santa Justa cuentan también con fuertes incrementos. Por su parte, San Bernardo ofrece un saldo positivo en todos los periodos comparados, ganando alrededor de 1.000 habitantes en 25 años, lo que motiva su división en dos secciones censales a partir de 2005. Otros casos que podrían resultar de interés son los de Doctor Barraquer, Barrio León, San José Obrero y El Fontanal, donde coincide un incremento de los indicadores sociales con el aumento de la población. Sin embargo, esto no sucede en todos los barrios del norte del centro histórico, donde el crecimiento es muy leve o incluso ligeramente negativo, algo aplicable también al caso de Triana.

Los sectores que reducen el envejecimiento, si bien de forma moderada, son escasos. Lo más destacable de la variación entre 1981 y 2006 es, sin duda, el rejuvenecimiento de ciertos barrios históricos de la ciudad. Es el caso de San Bernardo, donde la evolución de este indicador puede asociarse a la entrada de población en el sector durante el período de estudio. También es el caso de varios sectores dentro del centro histórico, sobre todo San Luis-Alameda, que sería la unidad espacial donde se ha producido la mayor reducción del envejecimiento (sin que le haya correspondido un incremento de su población).

Por último, respecto de la cuestión demográfica, la antigüedad de los hogares en 2001 es un indicador de gran interés. Conociendo los nuevos barrios que se han construido y habitado entre 1991 y 2001, sectores con valores atípicos para este dato, donde no hayan existido nuevos desarrollos, apuntarían a la existencia de una colonización de un sector consolidado, donde se estaría produciendo una invasiónsucesión (si bien el carácter de la misma no es identificable exclusivamente con base en esta variable).

En la Figura 5 se ha representado la proporción de hogares presentes en un barrio y que se han establecido en el mismo entre los censos de 1991 y 2001, con el objetivo de localizar casos atípicos en este sentido.

La representación cartográfica identifica claramente los barrios de colonización reciente, zonas que se han habitado fundamentalmente a partir de 1980. Sin embargo, el mayor interés en la distribución espacial de esta variable reside en los sectores próximos al centro con un dato elevado. Así, aparece un enclave de valores altos en el corredor Santa Justa-San Bernardo, justificado hasta cierto punto por un conjunto de nuevos desarrollos diseminados en los márgenes de los nuevos viarios o fruto del redesarrollo de amplias zonas incluidas en las unidades espaciales que lo componen. Destaca aquí San Bernardo, donde más de 55\% de los hogares censados en 2001 se estableció en la década de los noventa. 
FIGURA 5 | Hogares con menos de una década de antigüedad (porcentajes)

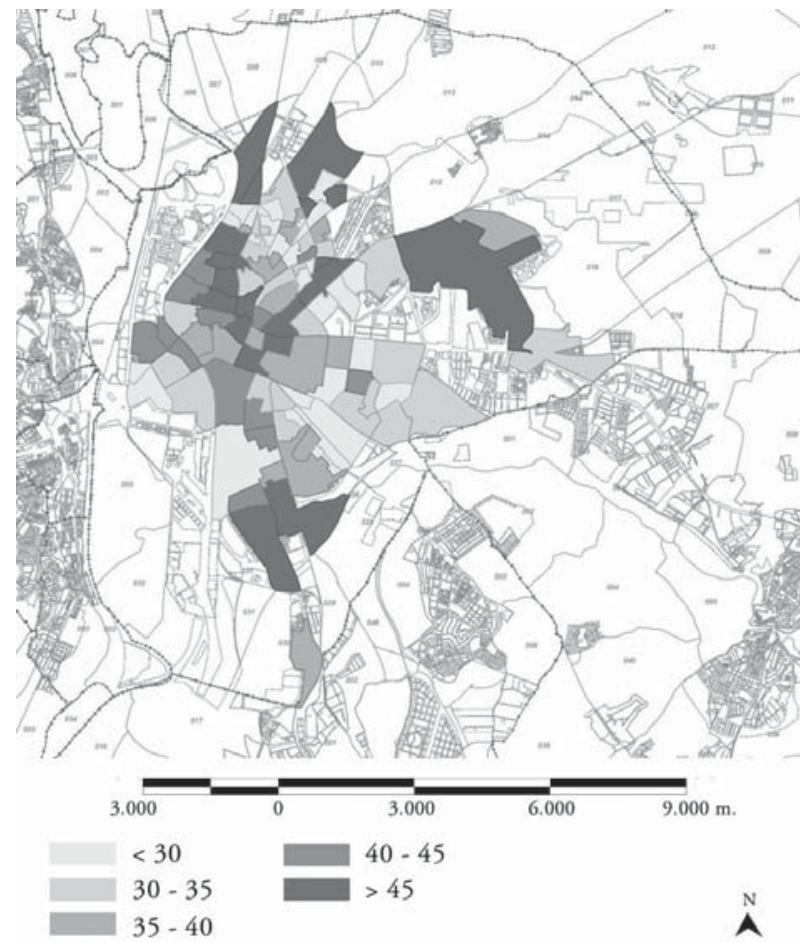

FUENTE ELABORACIÓN PROPIA A PARTIR DEL CENSO DE POBLACIÓN Y VIVIENDA 200 I

Otra aglomeración de nuevos hogares se localiza en el noreste del centro histórico, y a ellos se les une en extramuros Doctor Barraquer. Destacan en este caso San LuisAlameda y San Gil, con un $50 \%$ y un $48 \%$ de unidades con una antigüedad menor a diez ańos en el barrio para el censo de 2001.

\section{Estado de la edificación}

El volumen de edificios en estado deficiente, malo o ruinoso se ha reducido en términos generales para la ciudad de Sevilla. Todavía en 1981 existía una gran bolsa de edificaciones en mal estado, que alcanzaba un porcentaje considerable del total de viviendas y que, según la historiografía oficial, vendría dada en gran medida por la riada de 1961 y sus nefastos efectos sobre los sectores obreros del centro histórico y arrabales, así como por la ausencia de intervenciones rehabilitadoras posteriores.

En 1981 había una concentración evidente de viviendas degradadas, circunscrita a las barriadas en las que se sectoriza el viejo recinto amurallado. En él existía una clara diferencia entre su mitad norte y su mitad sur. Podrían incluso diferenciarse varias capas, una en el extremo norte, con los barrios de San Luis-Alameda, San Gil y San Lorenzo en un nivel extremo de degradación, con algo más de 40\% de su edificación en un estado deficiente. Una segunda franja identificable sería la del sur del centro histórico, con entre $20 \%$ y $30 \%$ de edificación en mal estado, igual tramo que San Julián. El resto del recinto intramuros contaba con bajos porcentajes, entre $20 \%$ y $30 \%$, de la edificación en estado deficiente, igual tramo que el extremo norte del arrabal de Triana y San Bernardo. Finalmente, desde San Bernardo hacia 
el norte se disponía una franja de unidades espaciales con su edificación bastante deteriorada, incluyendo La Florida, San Roque y La Calzada.

FIGURA 6 | Variación en el porcentaje de edificios en mal estado entre 1981 y 2001

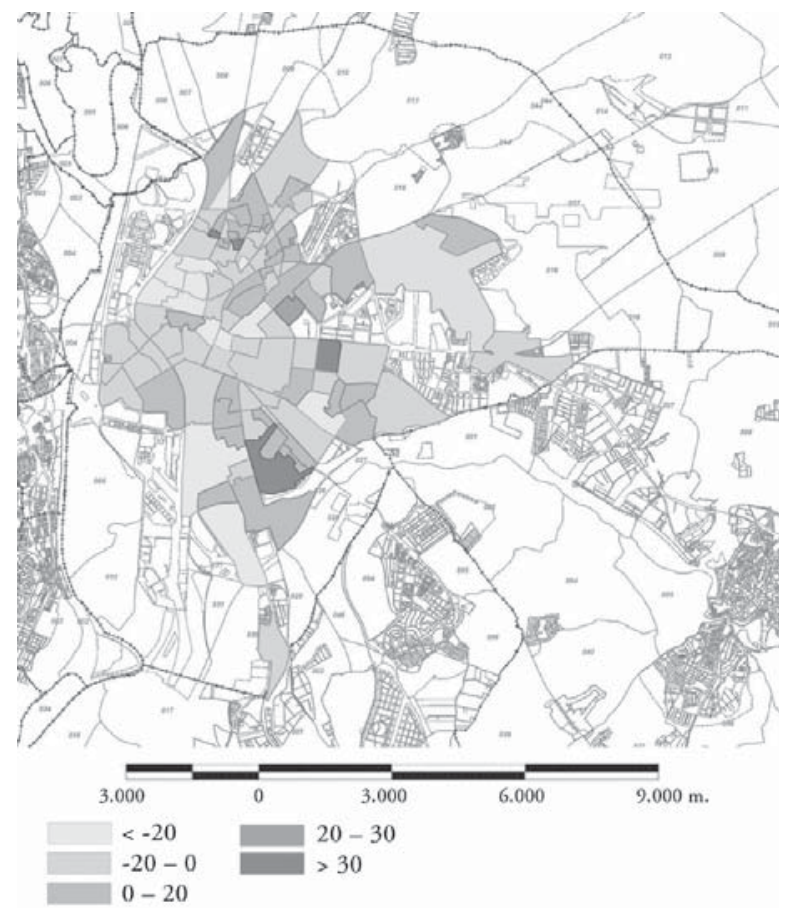

FUENTE ELABORACIÓN PROPIA A PARTIR DE LOS CENSOS DE POBLACIÓN Y VIVIENDA DE I98 I Y 200 I

En la Figura 6 se ha representado la variación en la proporción de viviendas en estado deficiente, malo o ruinoso para los barrios de Sevilla. Los hechos más reveladores son la notable reducción del indicador en el sector norte del recinto amurallado, con reducciones menores para Triana, San Roque y la mayor parte del resto del recinto intramuros. La regeneración también aparenta ser muy intensa en sectores como La Calzada y La Florida. En concreto, destaca San Luis-Alameda: a pesar de ser un barrio que seguía manteniendo en 2001 un gran número de edificaciones en estado deficiente, estas pasaron de constituir la mitad de toda la edificación a menos de 20\%. Reducciones similares se produjeron en San Gil o San Lorenzo. Por el contrario, sobresale el incremento del deterioro en algunas barriadas de la periferia obrera desarrollista de la ciudad, como Polígono Sur o Tres Barrios.

\section{Localización del acoso inmobiliario en Sevilla}

Sobre el plano catastral de Sevilla se han localizado los casos de acoso a inquilinos, expedientes abiertos por la Oficina Técnica de Atención al Inquilino en Situación de Abuso (Otainsa) entre 2004 y 2006, utilizándolos como indicador de la expulsión de inquilinos con bajos recursos. La vinculación del acoso inmobiliario al desplazamiento fruto de la gentrificación ya ha sido tratada en otros ámbitos (Díaz Parra, 2012). La 
representación cartográfica arroja pautas de distribución muy claras. En primer lugar, se hace evidente la concentración de los casos en el centro histórico de Sevilla y sus arrabales, mientras que el resto, una minoría, se localiza de forma dispersa por el espacio urbano.

La Figura 7 presenta, en principio, una clara concentración de situaciones de acoso en torno al centro histórico y Triana. Dentro de este gran conglomerado principal, las mayores concentraciones aparecen en el cuadrante noreste del centro histórico y en Triana, con una mayor dispersión de los casos en el cuadrante suroeste. A este primer anillo se le anexan los arrabales al este de la ciudad, San Bernardo, San Roque y La Calzada, de forma similar a sectores de colonización posterior, como La Florida o el barrio de autoconstrucción de principios del siglo xx de El Fontanal, al noreste, formando pequeńos conglomerados de importancia muy inferior a los que aparecen en el primer anillo. Se registran concentraciones similares en otros barrios de autoconstrucción de datación similar o algo posterior a El Fontanal, pero de carácter más periférico; es el caso del Barrio León.

\section{FIGURA 7 Localización de casos de acoso a inquilinos}

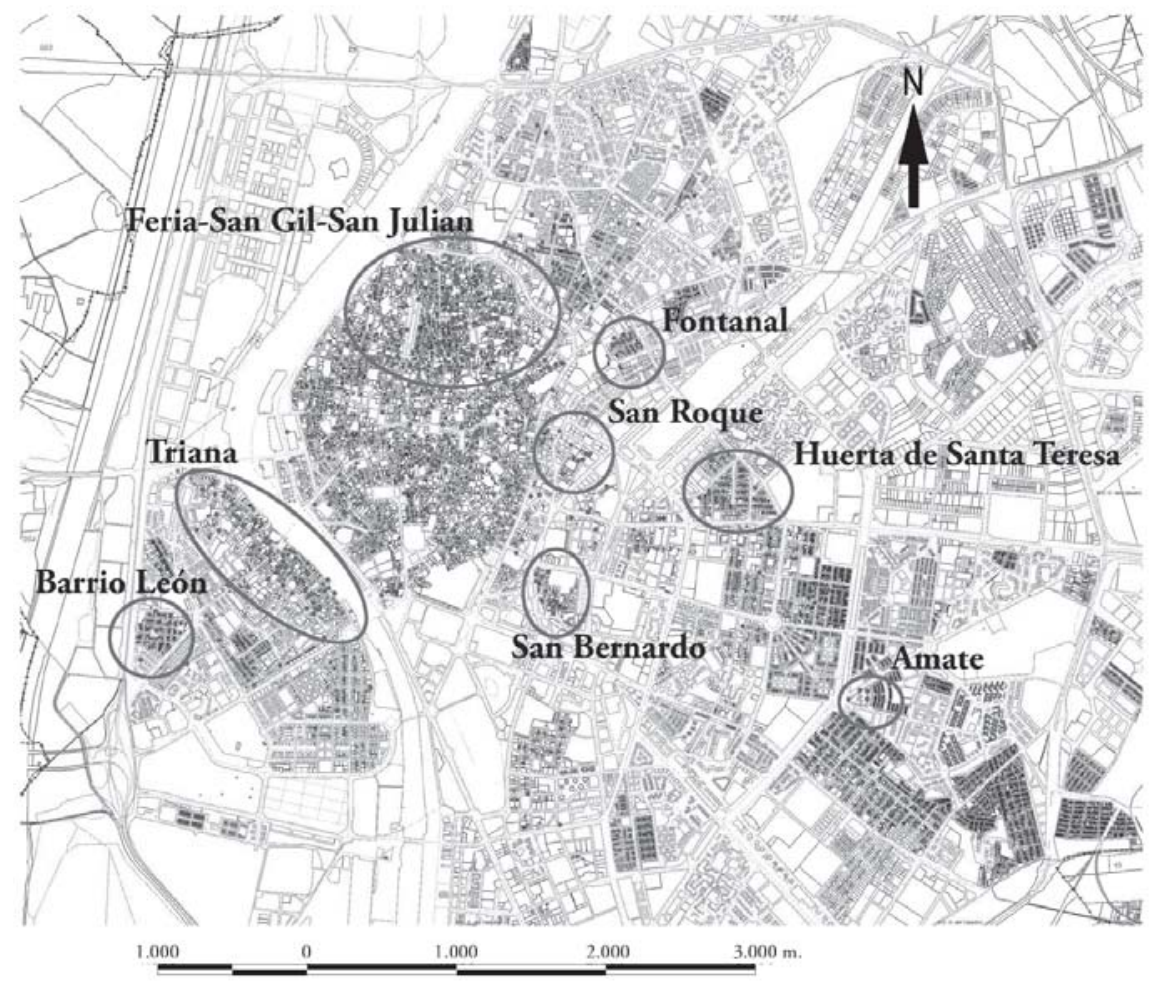

FUENTE ELABORACIÓN PROPIA A PARTIR DE OTAINSA

El antiguo recinto amurallado, sumado a los arrabales de La Calzada, Triana, San Bernardo y San Roque, supone cerca del 70\% de los casos. Triana representa un $11 \%$ y el centro histórico, un 51\%. De los diez barrios en los que se zonificó el centro, cerca del 60\% de los casos se ubica en tres de ellos, San Gil, San Julián y San Luis-Alameda (cuadrante noreste), que supondrían un 18\% del total. 


\section{La cambiante estructura socioespacial de la ciudad}

Los indicadores utilizados señalan los siguientes sectores como espacios donde ha acontecido una promoción social relativa, un inequívoco proceso de gentrificación, o existen indicios de este tipo de procesos:

- Frente de expansión sur de la ciudad: Aquí se encuentran dos grandes unidades espaciales, como son Los Bermejales y Pedro Salvador. El primero es, junto a Sevilla Este, el mayor nuevo desarrollo residencial de la ciudad en el contexto del PGOU de 1987, mientras que el segundo acoge gran parte de las nuevas construcciones en los márgenes de la avenida de Jerez. Se trata de sectores donde la promoción social relativa se explica fundamentalmente por la existencia de nuevas promociones residenciales, y donde no puede hablarse con seguridad de gentrificación.

- Cuadrante noreste del centro histórico: Es un sector en el que los indicadores apuntan a un claro proceso de gentrificación, aunque cabe señalar diferencias entre las unidades espaciales que lo componen. San Luis-Alameda parte en 1981 de una ratio sociolaboral de $58,17 \%$, lo que implica cerca del doble de trabajadores de cuello azul que profesionales y técnicos. En 2001 este dato había ascendido a $446 \%$, lo que implica que los profesionales y técnicos son ahora cuatro veces más que los trabajadores de cuello azul. Contaba con una de las mayores proporciones de edificaciones en estado deficiente, malo o ruinoso, superior al $40 \%$, valor que se reduce en veinte ańos a algo menos del $20 \%$, lo cual proporciona la segunda característica básica de la gentrificación. Esto coincide con dos datos especialmente reveladores: el hecho de ser el barrio con mayor incidencia del acoso a inquilinos con objetivo de desplazamiento, y ser aquel donde, en 2001, el 50\% de los hogares tenía una antigüedad inferior a diez años. Esto sin que haya existido un incremento de población durante el periodo de estudio. Por su parte, San Gil ofrece unos valores próximos y ligeramente inferiores a los de San Luis-Alameda para todos los indicadores expresados, con la diferencia de incrementar muy ligeramente su población. Ambos coinciden, además, en un notable rejuvenecimiento.

Por otro lado, los barrios del centro histórico contiguos a San Gil y San LuisAlameda no se deben descartar como parte de la gentrificación del centro norte. La principal razón para su exclusión de los sectores gentrificados es que en 1981 partían de una estratificación social moderadamente elevada (con la excepción de San Julián). Esta zona cuenta con valores extremadamente atípicos para el acoso inmobiliario y un dato similar a San Gil y San Luis-Alameda respecto de la antigüedad de los hogares en 2001: entre el 40\% y el 50\% establecido en los últimos diez años.

Con base en lo anterior, puede hablarse de una gentrificación del cuadrante noreste del centro histórico, con su epicentro en San Luis-Alameda y San Gil.

- Triana: Para el arrabal de Triana, todos los indicadores reflejan un proceso de gentrificación: promoción social relativa, rejuvenecimiento, elevada incidencia 
del acoso a inquilinos y elevadísima proporción de nuevos hogares para el censo de 2001. La única característica de la gentrificación matizable sería la menor reducción del número de viviendas en mal estado, menor que en San LuisAlameda o San Gil, aunque todavía relevante. Para todos estos indicadores, la unidad espacial que cubre el norte del arrabal presenta valores correspondientes a una mayor gentrificación que la mitad sur, que parte de valores sociales y de un estado de la vivienda manifiestamente mejores.

- Corredor Santa Justa-San Bernardo: Es necesario matizar que, debido a la compleja integración del ferrocarril en la ciudad, este espacio suponía (antes de la intervención en el marco del planeamiento general urbanístico de 1987-2006) un conjunto heterogéneo, compuesto de infraestructuras, fábricas y barriadas de diferente datación. Respecto de la cuestión de la gentrificación, la situación de estos barrios ha de ser tomada con precaución. Esto es así, debido a que los cambios producidos han estado necesariamente influidos por la existencia de nuevos desarrollos o redesarrollos residenciales. El caso más claro sería el de la unidad espacial denominada San José Obrero, que cubre la mayor parte del nuevo entorno residencial generado a partir de la construcción de la estación de Santa Justa, y donde la fuerte promoción social es el fruto evidente de las nuevas construcciones de elite sobre suelos recalificados y no de procesos de gentrificación de las barriadas preexistentes, las cuales son un conjunto de promociones de vivienda pública de corte funcionalista escasamente atractivas para grupos de altos ingresos.

El arrabal de La Calzada, por su parte, ha sido sustituido por nuevas promociones en fechas recientes, siendo una típica intervención de renovación urbana de corte desarrollista. Además, dicha operación ha sido ejecutada en gran parte sobre suelos que nunca estuvieron ocupados con anterioridad por edificación residencial. No obstante, esto no impide que se hayan producido procesos de gentrificación de nueva planta a una escala reducida.

El caso de San Bernardo es más polémico, ya que debe diferenciarse la unidad espacial definida como San Bernardo, y que cuenta desde 1981 con valores muy elevados de estratificación social, del arrabal histórico de San Bernardo. Este ocupa solo una pequeña porción de dicha unidad, separada del resto hasta el soterramiento del ferrocarril. Gracias a trabajos anteriores, se conoce que en la década de los ochenta predominaba en el arrabal una extracción social muy humilde, si bien mermada y envejecida (Fernández Salinas, 2003; y Vera, 1990).

San Bernardo es una de las unidades espaciales con mayor promoción social, intensa rehabilitación de la edificación, cierta concentración de casos de acoso a inquilinos y un 55\% de los hogares con menos de diez ańos de antigüedad en 2001. Al mismo tiempo, es un barrio que ha experimentado un notable incremento de población y un rejuvenecimiento de la misma. El problema reside en que los sectores que acoge esta unidad espacial son extremadamente variados, cubriendo desde el propio arrabal a los bloques de pisos de carácter burgués del Prado de San Sebastián, pasando por un buen número de nuevos desarrollos propiciados 
por la recalificación de antiguos terrenos de RENFE, la Red Nacional de Ferrocarriles Españoles, en fechas recientes. En este caso, un análisis cuantitativo requeriría trabajar a un nivel de desagregación de manzanas con la intención de identificar el cambio real producido.

- Doctor Barraquer: En Doctor Barraquer, por su parte, la promoción social se une a una elevada proporción de nuevos hogares, pero no es relevante el acoso a inquilinos ni una intensa intervención sobre el caserío preexistente. Por otro lado, este caso es similar al de San Bernardo, en la medida en que la mayor parte de la unidad espacial está ocupada por nuevos desarrollos residenciales de altas densidades sobre antiguos suelos asociados a la infraestructura del ferrocarril. Solo una pequeña parte del espacio corresponde al arrabal de la Macarena, que requeriría un análisis en profundidad para valorar si ha existido un proceso de gentrificación en el mismo.

- Otros sectores con una promoción social relativa notable han sido el barrio León y El Fontanal. Estos barrios reúnen una cantidad atípica, que no extremadamente atípica, de casos de acoso, y presentan una promoción social relativa similar a otros sectores gentrificados. En 1981 se encontraban muy por debajo de la media para el indicador de promoción social, pero en 2001 se habían situado por encima, lo cual podría explicarse en parte por un notable incremento de población en el periodo de estudio, por encima de los 4.000 individuos. Por otro lado, no hay signos de una intensa renovación del caserío, debido a que en estos barrios se partía de una edificación en buen estado; incluso han sufrido un cierto deterioro, y tampoco hay datos relevantes para la proporción de hogares nuevos, por debajo del 30\%. En ambos casos podemos hablar de la existencia de indicios de gentrificación.

\section{¿Procesos de gentrificación, repoblación o promoción social?}

Respecto de la metodología utilizada, el trabajo ha mostrado las limitaciones de una aproximación cuantitativa en función de fuentes estadísticas convencionales: padrón y censo. Se han encontrado dificultades generadas por el tamaño de las unidades espaciales elegidas, necesariamente grandes (agregaciones de secciones) para poder comparar los censos. El límite de desagregación deja fuera espacios de reducido tamańo, al tiempo que puede integrar en ocasiones sectores urbanos de un carácter social marcadamente diferenciado. Esto no desaconseja el uso del método sociodemográfico por unidades espaciales, pero exige localizar aquellos casos que escapan a un análisis cuantitativo y realizar un estudio en profundidad mediante otro tipo de técnicas. Así, se pone de relieve la necesidad de un tratamiento individualizado de los casos de gentrificación, que tienden a contar con distintos rangos temporales y, sobre todo, demandan metodologías diferenciadas. Es el caso del barrio sevillano de San Bernardo, donde cualquier aproximación estadística tendría que depender de métodos de encuesta, siendo los resultados de estas más que limitados por la práctica desaparición de los grupos de vecinos con mayor arraigo de la zona. En casos como este, sería más adecuado un estudio de la morfología urbana combinado con 
entrevistas cualitativas, si se fuese capaz de encontrar antiguos vecinos desplazados dispuestos a ser entrevistados.

A la hora de comparar las proposiciones que se hicieron al comienzo de este texto, observando el resultado del trabajo empírico, la discusión en torno a la existencia de procesos de gentrificación en Sevilla no es en ningún caso baladí. Puede afirmarse que en Sevilla hay espacios que se han "residencializado" y ha habido una promoción social considerable durante el periodo estudiado. Sin embargo, se ha demostrado que gran parte de las zonas obreras históricas han sufrido fuertes transformaciones, que coinciden con las características de un proceso de gentrificación y no con una repoblación o residencialización, y que tampoco están justificadas por la simple modificación de la estructura sociolaboral del conjunto de la población. Con base en esto, en primer lugar, puede descartarse la gentrificación como la expresión de la promoción social global de la ciudad. Diferentes sectores han evolucionado de diferente manera y solo unos pocos han variado notablemente su posición relativa en la estratificación socioespacial de la ciudad. Hay barrios que mantienen posiciones estables con valores relativamente altos o bajos respecto de indicadores sociales, así como otros que varían su posición relativa durante el marco temporal de referencia. A ellos apunta principalmente el interés de este trabajo. No obstante, la promoción social relativa no es suficiente para hablar de gentrificación. Resulta fundamental en este sentido la diferenciación realizada entre áreas con una promoción fruto de la colonización de nuevos vecindarios y la promoción social relativa no justificada por este extremo, donde es ineludible hablar de gentrificación. En segundo lugar, también debe descartarse la interpretación de la gentrificación como un proceso de repoblación. El cambio en los indicadores de la gentrificación viene vinculado a un fuerte incremento de la población en determinados ámbitos, pero no en otros. Los sectores en los que coincide el cambio social y urbanístico con los mayores incrementos del número de habitantes, son barrios en los que existen nuevos desarrollos residenciales fruto de la recalificación de terrenos. Otras unidades espaciales donde los indicadores señalan la existencia de un obvio proceso de promoción social apenas han variado su stock de población -caso de San LuisAlameda y Triana-, coincidiendo además con una fuerte concentración de casos de acoso a inquilinos con objeto de desplazamiento. Así, se comprueba que en ciertos barrios existe promoción social y mejora de la edificación que no es explicable más que por la entrada de nueva población con elevados recursos y salida de población de perfil humilde (dentro de la cual ha tenido cierto peso el empleo de tácticas criminales por parte de los propietarios de viviendas en alquiler), confirmándose así la proposición de partida de este trabajo.

La responsabilidad de la intervención pública en cualquiera de los procesos sociales que recientemente se han desarrollado en los lugares centrales de Sevilla es ineludible. Del conjunto de transformaciones (en muchos casos positivas) que estos han sufrido, aspectos como el acoso inmobiliario, el desplazamiento de población y la sustitución social han quedado en evidencia. Si bien los medios desplegados para regenerar los sectores centrales degradados (y no los ubicados en localizaciones periféricas) fueron muy generosos en su momento, las iniciativas para corregir o atenuar las mencionadas consecuencias negativas han sido inexistentes o muy tardías. Un 
análisis en profundidad de estas medidas, en especial la contribución de la propia Otainsa, excede los objetivos de este trabajo. En cualquier caso, sería deseable que en futuras intervenciones se tuviera en cuenta en mayor medida el potencial impacto social y no solo las posibilidades de regeneración urbanística y dinamización económica de la intervención sobre los espacios centrales de la ciudad.

\section{Referencias bibliográficas}

Atkinson, R. (2000). Measuring gentrification and displacement in greater London. Urban Studies, 37(1), 149-165. doi: 10.1080/0042098002339

Beauregard, R. (1986). The chaos and complexity of gentrification. En N. Smith \& P. Williams (Eds.), Gentrification of the City (pp. 35-55). Boston / Londres: Unwin Hyman.

Bromley, R. D. F., Tallon, A. R. \& Thomas, C. J. (2005). City centre regeneration through residential development: contributing to sustainability. Urban Studies, 42(13), 24072429. doi: $10.1080 / 00420980500379537$

Butler, T. (2007). For gentrification? Environment and Planning A, 39(1), 162-181.

Cruz Villalón, J. (Coord.). (1986). La población de Sevilla. Sevilla: Servicio de publicaciones del Ayuntamiento de Sevilla.

Davison, M. \& Lees, L. (2010). New-build gentrification: Its histories, trajectories, and critical geographies. Population, Space and Place ${ }_{s}$ 1(16), 395-411. doi: 10.1002/psp.584

Díaz Parra, I. (2012). Desplazamiento, acoso inmobiliario y espacio gentrificable en el caso de Sevilla. Encrucijadas. Revista crítica de Ciencias Sociales, 1(2), 48-68. En http://www. encrucijadas.org/2011/12/diaz-parra-n2.html

Fernández Salinas, V. (1993). Las grandes transformaciones urbanas de Sevilla durante los años previos a la exposición universal. Estudios Geográficos, 54(212), 387-407.

Fernández Salinas, V. (2003). La vivienda modesta y patrimonio cultural: los corrales y patios de vecindad en el conjunto histórico de Sevilla. Scripta Nova. Revista electrónica de Geografía y Ciencias Sociales, 6(146). En http://www.ub.edu/geocrit/sn/sn-146(070).htm

Freeman, L. (2008). Comment on 'The eviction of critical perspectives from gentrification research'. International Jounal of Urban and Regional Research, 32(1), 186-191. doi: 10.1111/j.1468-2427.2008.00771.x

García Bernal, J. (Coord.). (2005). La ciudad y su gente. Historia urbana de Sevilla. Sevilla: Escuela Libre de Historiadores.

García Jaén, P. (1998). Aplicaciones de la iniciativa comunitaria. Urban. Boletín de la Asociación de Geógrafos Españoles, 12(26), 191-206. En dialnet.unirioja.es/descarga/ articulo/1318638.pdf

Gerencia de Urbanismo (1987). Plan General de Ordenación Urbana 1987. Memoria de propuestas. Sevilla: Ayuntamiento de Sevilla.

Gerencia de Urbanismo. (1994). Avance del Plan Especial de Protección del Conjunto Histórico de Sevilla. Sevilla: Ayuntamiento de Sevilla.

González Dorado, A. (1975). Sevilla: centralidad regional y organización interna de su espacio. Madrid: Moneda y Crédito. 
Hamnett, C. (2003). Gentrification and the middle-class remaking of inner London, 19612001. Urban Studies, 4O(12), 2401-2426. doi: 10.1080/0042098032000136138

Hamnett, C. (2009). The new Mikado? Tom Slater, gentrification and displacement. City, 13(4), 476-482. doi: 10.1080/13604810903298672

Harvey, D. (1977). Urbanismo y desigualdad social. México: Siglo xxi.

Lees, L., Slater, T. \& Wyly, E. (2008). Gentrification. Londres: Routledge.

Ley, D. (1983). A social geography of the city. Nueva York: Harper and Row.

Ley, D. (1996). The new middle classes and the remaking of the central city. Oxford: Oxford University Press.

Marín de Terán, L. (1980). Sevilla, centro urbano y barriadas. Sevilla: Servicio de Publicaciones del Ayuntamiento de Sevilla.

Martín García, A. (1996). Sevilla (1872-1994) ciudad y territorio: de lo local a lo metropolitano. Sevilla: Fundación Cultural Colegio Oficial de Aparejadores y Arquitectos Técnicos.

Millner, J. C. (2003). El salario ideal. La teoría de las clases y de la cultura en el siglo XX. Barcelona: Gedisa.

Sabatina, F., Sarella, M. \& Vásquez, H. (2012). Gentrificación sin expulsión o la ciudad latinoamericana en una encrucijada histórica. Arquitectura_arte_diseño, 180. En http://www.revista180.udp.cl/ediciones/24/gentrificacion_24.htm

Slater, T. (2006). The eviction of critical perspectives from gentrification research. International Journal of Urban and Regional Research, 30(4), 737-757. doi: 10.1111/j.14682427.2006.00689.x

Slater, T. (2009). Missing Marcuse. On gentrification and displacement. City, 13(2-3), 293311. doi: 10.1080/13604810902982250

Smith, N. \& Williams, P. (1986). Gentrification of the city. Boston: Unwin Hyman.

Smith, N. (1982). Gentrification and uneven development. Economic Geography, 58, 139-155.

Smith, N. (1996). The new urban frontier. Gentrification and the revanchist city. Oxford: Routledge.

Vera, A. L. (1990). San Bernardo, génesis y decadencia demográfica de un barrio sevillano. En J. Cosano Moyano (Dir.), Actas del VIII Congreso de Profesores Investigadores, celebrado en Baena del 11 al 13 de septiembre de 1989. Baena: Ayuntamiento de Baena / Hespérides.

Wacquant, L. (2008). Relocating gentrification: The working class, science and the state in recent urban research. International Journal of Urban and Regional Research, 32(1), 198-205. doi: 10.1111/j.1468-2427.2008.00774.x 